\title{
Delamination Testing of AlSi10Mg Sandwich Structures with Pyramidal Lattice Truss Core made by Laser Powder Bed Fusion
}

\author{
M. Nuño ${ }^{\dagger}$, J. Bühring ${ }^{*}{ }^{\dagger}$, M. N. Rao and K. -U. Schröder
}

\begin{abstract}
Sandwich structures possess a high bending stiffness compared to monolithic structures with a similar weight. This makes them very suitable for lightweight applications, where high stiffness to weight ratios are needed. Most common manufacturing methods of sandwich structures involve adhesive bonding of the core material with the sheets. However, adhesive bonding is prone to delamination, a failure mode that is often difficult to detect. This paper presents the results of delamination testing of fully additive manufactured (AM) AISi10Mg sandwich structures with pyramidal lattice truss core using Laser Powder Bed Fusion (LPBF). The faces and struts are $0.5 \mathrm{~mm}$ thick, while the core is $2 \mathrm{~mm}$ thick. The inclination of the struts is $45^{\circ}$. To characterise the bonding strength, climbing drum peel tests and out-of-plane tensile tests are performed. Analytical formulas are derived to predict the expected failure loads and modes. The analytics and tests are supported by finite element (FE) calculations. From the analytic approach, design guidelines to avoid delamination in AM sandwich structures are derived. The study presents a critical face sheet thickness to strut diameter ratio for which the structure can delaminate. This ratio is mainly influenced by the inclination of the struts. The peel tests resulted in face yielding, which can also be inferred from the analytics and numerics. The out-of-plane tensile tests didn't damage the structure.
\end{abstract}

Keywords: Additive Manufacturing, Sandwich Structures, Pyramidal Lattice Core

\section{Introduction}

During the last years, the usage of light metals in highly complex structures has come into focus due to the progressive development in additive manufacturing technology (AM). Innovative processes, such as Laser Powder Bed Fusion (LPBF), Electron Beam Melting (EBM) or Laser Metal Deposition (LMD), make it possible to manufacture geometries that would not be producible with conventional methods. The range of materials is now so large that a suitable alloy is available for almost every application. Aluminium, titanium or magnesium alloys are particularly interesting for lightweight applications

\footnotetext{
*Correspondence: jannik.buehring@sla.rwth-aachen.de ${ }^{\dagger} \mathrm{M}$. Nuño and J. Bühring contributed equally as first authors Institute of Structural Mechanics and Lightweight Design, RWTH Aachen University, Wüllnerstraße 7, 52072 Aachen, Germany
}

in aerospace [1-9], automotive [10-12] or biomechanics [13-17], as they provide good relations in terms of strength, stiffness and weight, and provide further application-specific properties i.e. regarding thermal or corrosive requirements. An often used aluminium alloy for LPBF is AlSi10Mg (Al-based alloy with 10wt\% Si and $<1 \mathrm{wt} \% \mathrm{Mg})$ as it provides a good processability $[18,19]$ and furthermore good thermal $[20,21]$ and mechanical properties [18, 22, 23].

One of the most weight-efficient structures for lightweight applications is a sandwich. Sandwich structures usually consist of two thin face sheets, which are adhesively connected to a much thicker core structure, leading to outstanding bending and buckling properties. A lot of different core structures and materials for sandwich applications have been developed during the past years [24]. However, the most commonly used are honeycombs 
and foams. Even if the structural advantages outweigh the disadvantages, delamination is a significant problem for sandwich structures. Often the adhesive bonding is responsible for the failure of sandwich structures and can in many cases be traced back to delamination, which can occur due to local damages of the adhesive connection or local buckling of the face sheets. Furthermore, especially in the case of honeycomb core structures, the bonding surface is small and, therefore, also the maximum transferable load. To increase the bonding strength, an increase of the bonding surface would be reasonable. But, whereas the specific weight is proportional to the vertical tensile module and the tensile strength, the peeling resistance of the skin/core bonding is not exceedable.

With the establishment of new manufacturing processes, such as additive manufacturing, alternative approaches for sandwich structures get attractive. By using lattice structures for the sandwich core, the sandwich can be printed as a single part. Thus, a direct connection between the core and the faces is established. Furthermore, lattice structures offer a lot of design parameters, which allows for fully application-specific designs with customized core properties.

First fundamental investigations about sandwich structures with lattice cores were presented by Wicks \& Hutchinson $[25,26]$. They show that truss core sandwich structures have high flexibility in designing the structural behaviour. Compared to honeycomb structures, much better structural properties against bending and compression loads, regarding stiffness or strength, can be achieved. For cellular structures, Gibson \& Ashby [27, 28] show basic formulations for the mechanical properties of lattice structures as a function of the relative cell density. They show that different elastic properties of sandwich structures scale linearly with the relative density of the core. For sandwich structures with tetrahedral and pyramidal core under 3-point bending, Deshpande \& Fleck $[29,30]$ derived equivalent expressions. The results are extended by the research of Evans et al. [31, 32] and Chiras et al. [33] for shear and compression loads. Zok et al. [34] show that the main failure mechanisms of sandwich panels with a pyramidal truss core are: face sheet yielding, face sheet buckling, core member yielding and core member buckling.

Basic studies about failure modes of sandwich structures have been done, especially by Zenkert et al. [35-37]. They show that sandwich beams under quasi-static and fatigue bending loads tend to delaminate for the most tested configurations. Some research focuses on methods to stop these delaminations instead of avoiding them. For example, Grenstedt [38, 39] implemented peel stoppers during manufacturing. Other approaches focus on the creation of sandwich structures with high transverse stiffness and strength $[40,41]$ to avoid delaminations. Furthermore, Jakobsen et al. [42] show an alternative approach. They reroute the delamination to confine it to a predefined zone in the sandwich. By this, they are able to stop the propagation of the delamination. According to the author's knowledge, no delamination studies have been performed to date on fully AM sandwich structures with lattice core. Since the delamination behaviour of such structures has not been investigated, this study focuses on a specific geometry.

This research's main objective is to develop simple criteria for avoiding delamination in AM sandwich structures with pyramidal cores. For this, their bonding strength, and delamination resistance are investigated. Analytical preliminary considerations regarding the peeling strength of sandwich structures with lattice core are supported by FE studies and physical tests. Figure 1 shows the considered AM sandwich structure.

\section{Methods and Materials}

\subsection{Materials and Specimens}

All structures were made of AlSi10Mg via LPBF by an external manufacturer using an EOS M290 printer with $30 \mu \mathrm{m}$ layer thickness. No special post-processing and no heat treatment were used for the specimens. The nominal geometric parameters of the structures are summed up in Table 1. Due to manufacturing restrictions, a face sheet thickness $t_{f}=0.5 \mathrm{~mm}$, a core thickness $t_{c}=2 \mathrm{~mm}$, a strut diameter of $d=0.5 \mathrm{~mm}$ and a relative strut angle of $\omega=45^{\circ}$ were used, resulting in a total sandwich height of $h=3 \mathrm{~mm}$. The radius of the struts is defined using $r$. Figure 2 shows a schematic sketch of a lattice unit cell with its face sheet section. To describe the structural properties of the lattice core, simple calculation methods can be used for strength and stiffness. The in-plane Young's modulus $E_{11}=E_{22}=E_{s}$ and shear modulus $G_{12}=G_{s}$ in 1-direction and 2-direction are dominated by the face sheets. The Young's modulus $E_{s}$ and shear modulus $G_{s}$ of the solid material are used. The core influence is neglected since, according to the classic sandwich

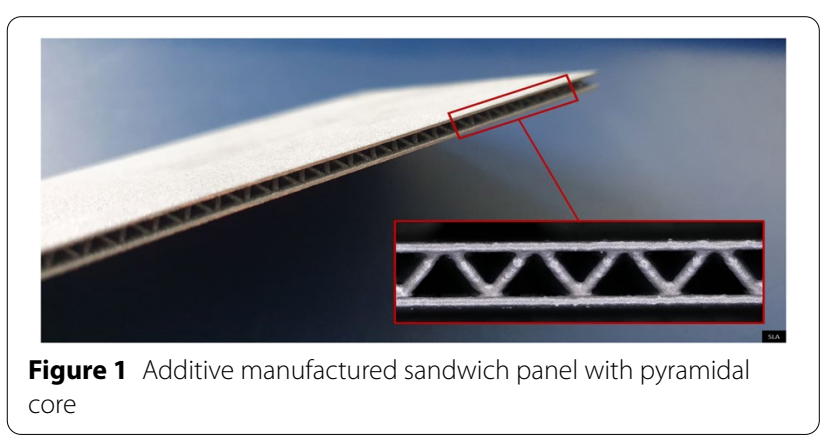



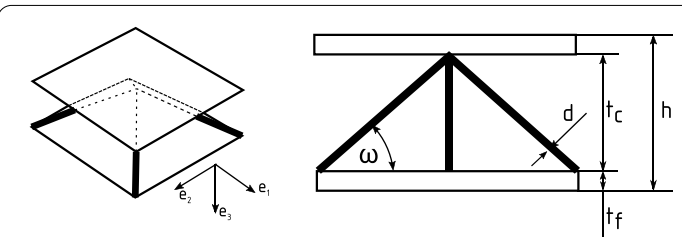

Figure 2 Unit cell of sandwich structures with geometric parameters

Table 1 Nominal parameters of the considered AM sandwich structures

\begin{tabular}{llll}
\hline $\boldsymbol{t}_{\boldsymbol{c}}$ & $\boldsymbol{r}$ & $\boldsymbol{\omega}$ & $\boldsymbol{t}_{\boldsymbol{f}}$ \\
\hline $2 \mathrm{~mm}$ & $0.25 \mathrm{~mm}$ & $45^{\circ}$ & $0.5 \mathrm{~mm}$
\end{tabular}

theory, the influence on the elastic constants is small. Effective elastic properties of lattice structures are usually defined as a function of the dimensionless relative density $\bar{\rho}$, which defines the ratio between the struts mass and the mass of the enclosing equivalent solid mass using the same material. For pyramidal cores, the relative density can be calculated with Eq. (1):

$$
\bar{\rho}=\frac{\rho}{\rho_{s}}=\frac{4 \pi r^{2} l}{b^{2} t_{c}}=\frac{2 \pi \tan (\omega)}{\cos (\omega)}\left(\frac{r}{t_{c}}\right)^{2} .
$$

Equations for the out-of-plane Young's modulus and shear modulus can be derived using the stiffness method, assuming pinned struts as shown for example in Ref. [29]:

$$
\begin{aligned}
& E_{33}=E_{s} \bar{\rho} \sin (\omega)^{4}, \\
& G_{13}=G_{23}=\frac{1}{8} E_{s} \bar{\rho} \sin (2 \omega)^{2} .
\end{aligned}
$$

Due to the overlapping of the struts, the bonding surface between the core and the face sheets cannot be assumed to be circular, as shown in Figure 3. Since no nondestructive measuring method is accessible, the nominal bonding surface is extracted from the CAD model, which was used for the manufacturing, and derived analytically using the following assumption:

$$
A_{c}=d^{2} \arctan (\csc \omega) \csc \omega .
$$

Both solutions result in a cross-section of the connection double-point of $A_{c} \approx 0.34 \mathrm{~mm}^{2}$ for the nominal strut diameter of $d=0.5 \mathrm{~mm}$.

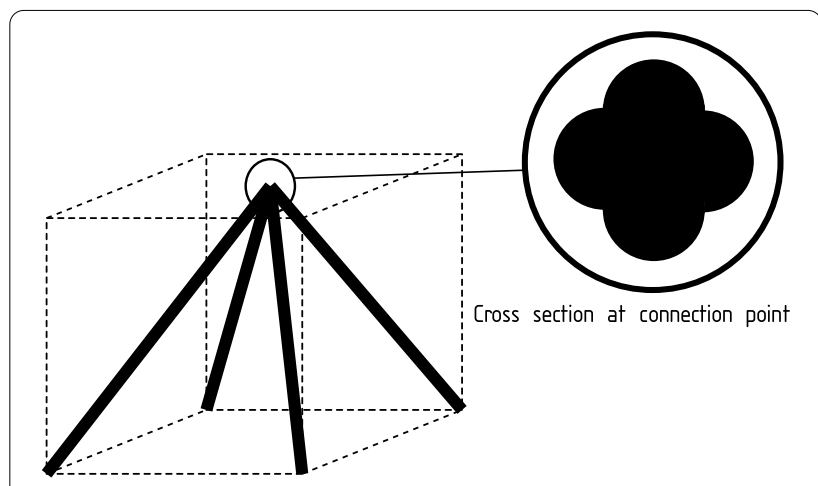

Figure 3 Non-circular connection between face-sheets and lattice core structure: $A_{c} \approx 0.34 \mathrm{~mm}^{2}$

Material properties of AM structures vary depending on used machine parameters while manufacturing. Since the results are very sensitive to stiffness and strength properties, the material properties for the calculations were determined by doing tensile tests. The specimens were produced from AM sandwich structures by carefully separating the face sheets from the core structure and afterwards milling them to a bone shape. A total number of three specimens was tested. All specimens were tested under the same conditions with displacement controlled load introduction and $\dot{u}=0.5 \mathrm{~mm} / \mathrm{min}$.

\subsection{Experimental Set-Up}

\subsubsection{Out-of-Plane Tensile Test}

A total number of 3 specimens with 25 unit cells $(5 \times 5)$ is tested. The tests are performed quasi-static and displacement controlled with $\dot{u}=0.2 \mathrm{~mm} / \mathrm{min}$, according to DIN 53292 on an Instron 5567 electric universal testing machine. To neglect influences resulting from clamping torque, a moment-free fixture, which equals a cardanic bearing, is constructed (Figure 4).

The face sheets of the specimens are adhesively bonded with the bearings. A 3M Scotch-Weld EC-9323-2 high strength epoxy adhesive is used. The manufacturer specifies the adhesive strength to be $29 \mathrm{MPa}$, resulting in a higher maximum load than the expected strength of the connection between the lattice core and the face sheets. The specimens are cut from large AM plates using a band saw. Due to these circumstances, the outer geometry of the specimens varies and can be found in Table 2. Furthermore, the resulting maximum allowable forces, before the strength of 


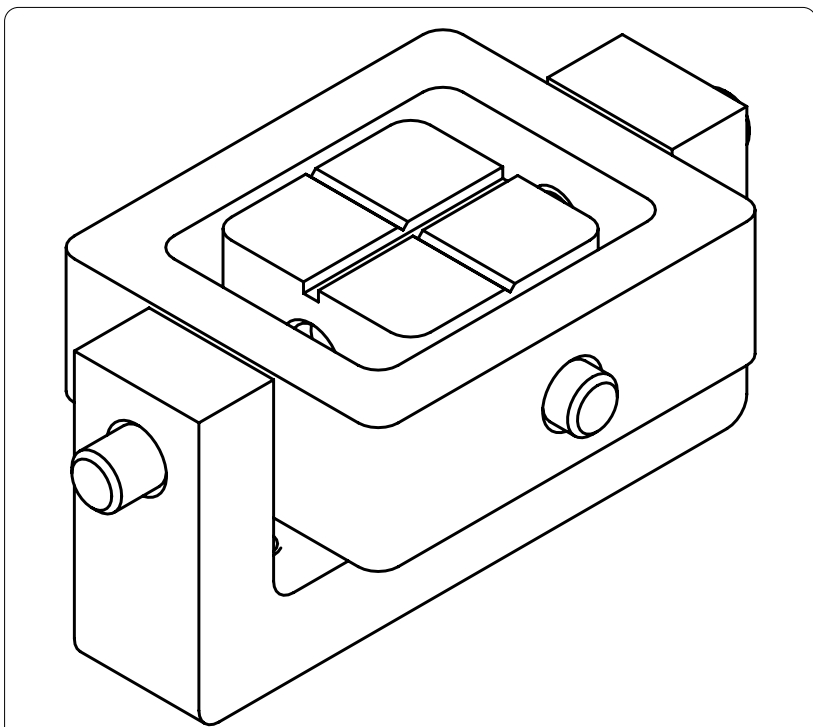

Figure 4 Cardanic bearing for out-of-plane tensile tests

the adhesive would be exceeded, are shown. All these force are much higher in comparison to the binding strength between face sheets and core, assuming the lowest ultimate strength of $R_{m} \approx 250 \mathrm{MPa}$, determined from the face sheet tensile tests (see 10 for reference), which results in a maximum force of $F_{\max \text {, core }}=2.125$ $\mathrm{kN}$. Since the exact ultimate strength is unknown, the expected failure loads of the three specimens should

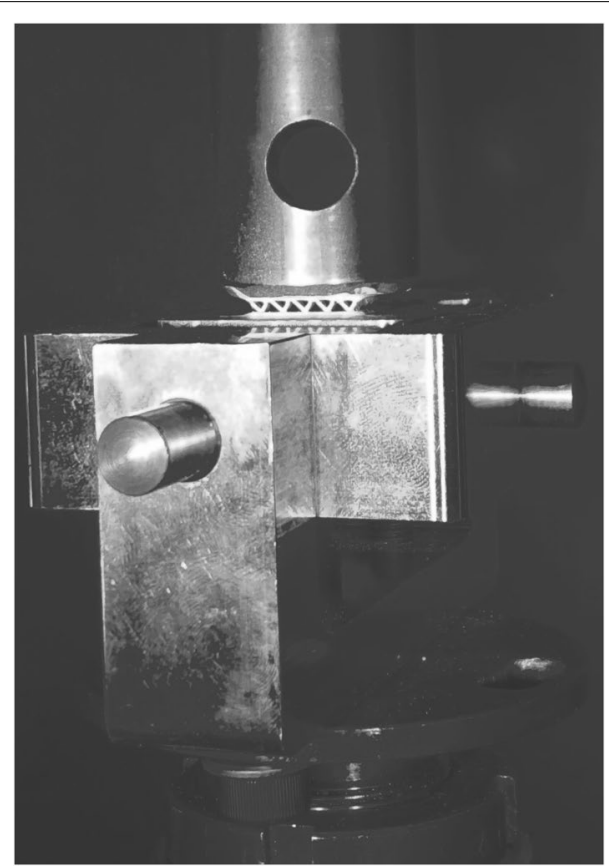

Figure $\mathbf{5}$ Final test set-up for out-of-plane tensile tests be in the range of $2-3 \mathrm{kN}$, assuming that the load is evenly distributed on the connection double-points and the plastic stress distribution is homogeneous. The final test set-up is shown in Figure 5. The displacement of the machine, as well as the force in the load cell, are documented. Additionally, the tests are monitored with a DIC technique, using a GOM Aramis 4M system, which allows measuring three-dimensional displacements and strain information of a surface by measuring absolute and relative multiple double-point movements.

\subsubsection{Climbing Drum Peel Test}

The climbing drum peel test is a method to determine the resistance of bonded sandwich cores against peeling forces acting perpendicular to the surface layer. The drum transforms the vertical force of the testing machine into a moment so that the connection between the face sheets and the core is directly loaded. The tests are done according to DIN 53295. The most important values are the diameter of the drum itself and the flange, where the specimens are fixed. The difference between both results in a translation of the tension axis. By this, a moment in the midpoint of the drum results, which has to be carried by the specimen. If the stresses get higher than the critical stress, the face sheets will delaminate. This value is known as specific peel moment and can be calculated according to DIN 53295 as follows:

$$
M_{\text {peel }}=\frac{\left(F_{p}-F_{1}\right)\left(r_{f}-r_{d}\right)}{b} .
$$

The force $F_{1}$ is the force needed to deform the face sheet itself and has to be substracted from the peelforce $F_{p}$. The flange radius is $r_{f}, r_{i}$ the drum radius and $b$ the width of the specimen. The flange radius can be calculated from the diameter of the drum $d_{d}$ and the thickness $a$ of the steel strip, which is needed to introduce the machine load into the peel fixture. It results in $r_{f}=\left(d_{f}+a\right) / 2=62.9 \mathrm{~mm}$, since $d_{f}=99 \mathrm{~mm}$ and $a=0.8 \mathrm{~mm}$. The drum radius $r_{d}$ can be calculated from the drum diameter $d_{d}=99 \mathrm{~mm}$ and the thickness of the face sheet $t_{f}=0.5 \mathrm{~mm}$ and results in $r_{i}=\left(d_{d}+t_{f}\right) / 2=49.8 \mathrm{~mm}$. The test set-up is slightly changed in comparison to the standard, but the impact

Table 2 Nominal parameters of AM sandwich structures

\begin{tabular}{llll}
\hline & Specimen 1 & Specimen 2 & Specimen 3 \\
\hline Dim. $\left(\mathrm{mm}^{2}\right)$ & $17.6 \times 14.6$ & $17.5 \times 15.5$ & $17.1 \times 18.0$ \\
Area $\left(\mathrm{mm}^{2}\right)$ & 257 & 271 & 308 \\
Force $(\mathrm{kN})$ & 7.45 & 7.86 & 8.93 \\
\hline
\end{tabular}




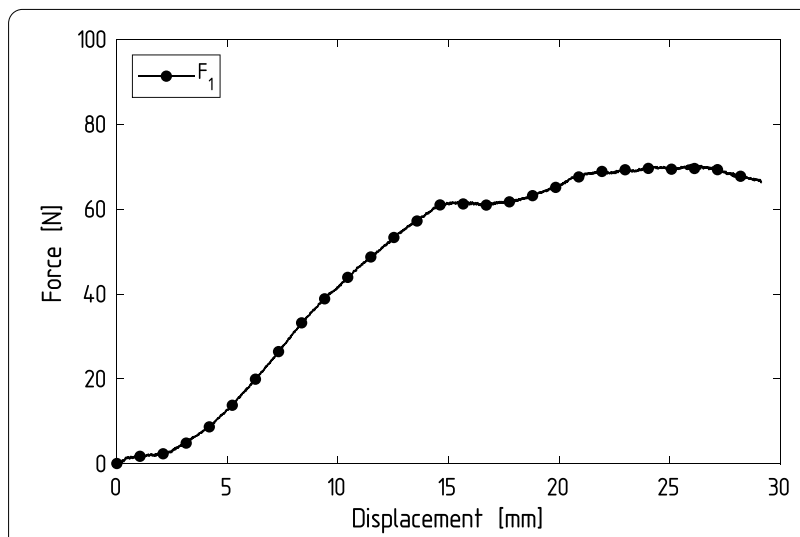

Figure 6 Preliminary peel test on a face sheet to extract $F_{1}$

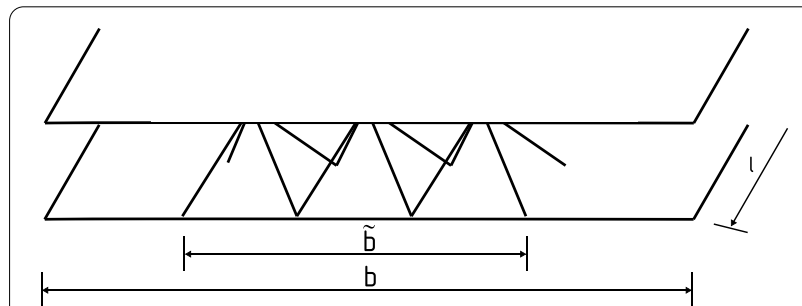

Figure 7 Schematic sketch of the peel test specimens

on the calculated resulting peel moment is small, since the relative error results in $\epsilon_{\text {rel }} \approx 1.6 \%$, which is a tolerable value. The force $F_{1}$ is determined by a preliminary test with a face sheet only. The specimen is produced by carefully cutting a face sheet from a sandwich structure. $F_{1}$ consists of a part which is needed to position the peel fixture $F_{\text {pos }}$ and a part which is needed to deform, or rather roll the face sheet $F_{\text {roll }}$. Figure 6 shows the result of the test to extract $F_{1}$. During the first $15 \mathrm{~mm}$ of displacement, the drum and steel strips get loaded and aligned. The followed plateau at $15 \mathrm{~mm}$ defines the needed force $F_{\text {pos. }}$. The additionally needed force to reach the second plateau at $25 \mathrm{~mm}$ defines $F_{\text {roll }}$, which lead in sum to $F_{1}=69.31 \mathrm{~N}$.

Regarding the climbing drum peel tests, a total number of 3 specimens is tested. In Table 3 the specific geometric parameters are summed up. Furthermore Figure 7 shows a sketch of the specimens and Table 4 the resulting expected relevant cross-sections. $R_{m}=350 \mathrm{MPa}$ is used. The number of cells $n$ was successively decreased since no delamination could be incited with high cell numbers. Figure 8 shows the final test set-up. All drum peel tests are done displacement controlled using $\dot{u}=25 \mathrm{~mm} / \mathrm{min}$. The load from the load cell and the displacement are documented during the tests.

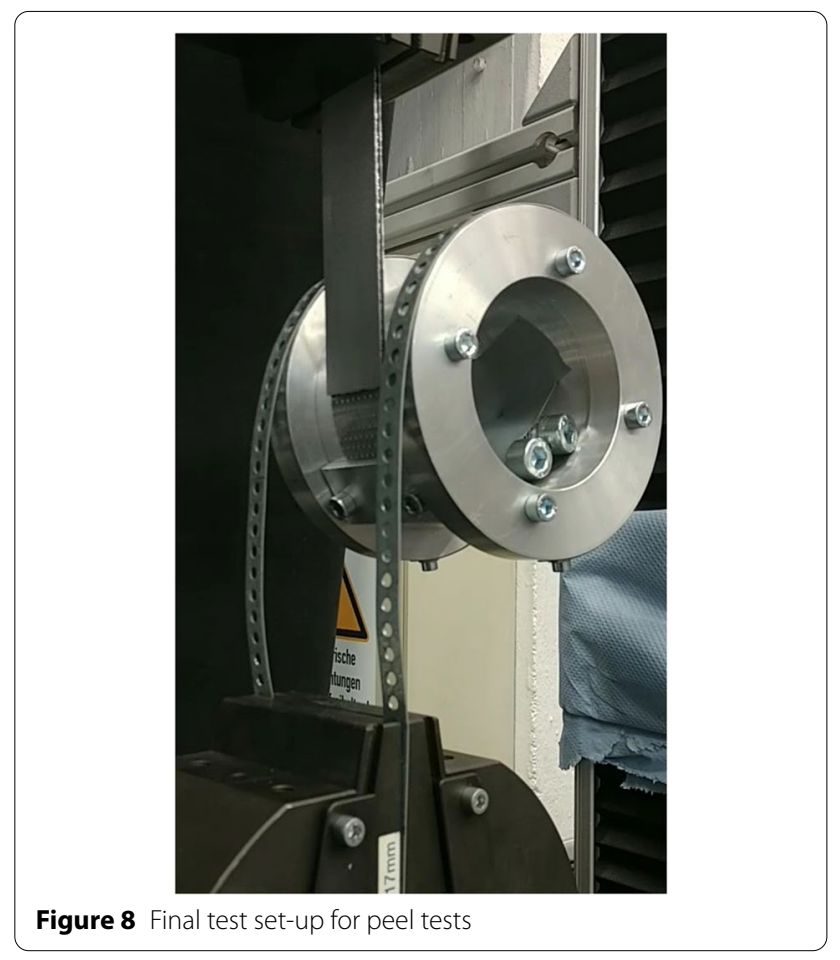

Table 3 Geometric paramters of specimens for climbing drum peel test

\begin{tabular}{llll}
\hline & Specimen $\mathbf{1}$ & Specimen 2 & Specimen $\mathbf{3}$ \\
\hline$n$ & 9 & 5 & 2 \\
$b(\mathrm{~mm})$ & 33.4 & 35.1 & 30.1 \\
$\tilde{b}(\mathrm{~mm})$ & 27.9 & 15.5 & 6.2 \\
$I(\mathrm{~mm})$ & 150 & 150 & 150 \\
\hline
\end{tabular}

Table 4 Resulting expected cross sectional areas for drum peel test

\begin{tabular}{llll}
\hline & Specimen 1 & Specimen 2 & Specimen 3 \\
\hline$A_{b}\left(\mathrm{~mm}^{2}\right)$ & 0.34 & 0.34 & 0.34 \\
$A_{s}\left(\mathrm{~mm}^{2}\right)$ & 0.19 & 0.19 & 0.19 \\
$A_{f}\left(\mathrm{~mm}^{2}\right)$ & 16.7 & 17.55 & 15.05 \\
\hline
\end{tabular}

\subsection{Finite Element Model}

To validate the derived formulas for the failure modes and to compare the results with the experiments, static FE simulations are performed. The preprocessing and calculations are performed with Abaqus/Standard. For each calculation, a convergence study is done. All models are meshed with 10-node quadratic tetrahedral elements with improved surface stress visualisation 


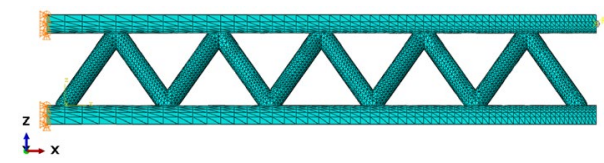

(a) Peel test with the geometry of the test specimens

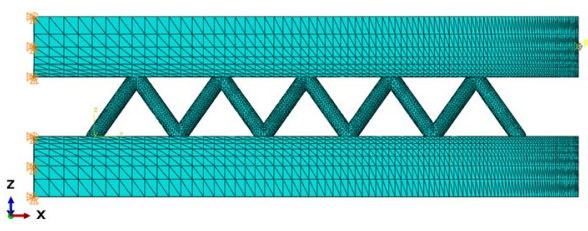

(b) Peel test with thicker faces

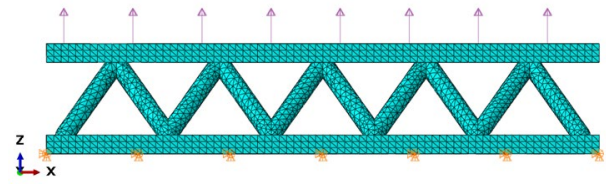

(c) Out-of-plane tensile test

Figure 9 Finite element models for the peel and out-of-plane tensile test

(Abaqus element C3D10HS). The models used for the peel test simulations are shown in Figure 9a and b. One side of the sandwich is pinned (top and bottom face sheet edges). On the opposite side, a bending moment is applied using a multiple point constraint (MPC) to all nodes on the top sheet. The mesh in the vicinity of the critical connection area is refined using a seed of $0.05 \mathrm{~mm}$. In the areas close to the support, where low stresses are expected, the seed used is $0.5 \mathrm{~mm}$. The FE model simulating the out-of-plane tension is shown in Figure 9c. The bottom nodes of the bottom sheet are pinned, and a homogeneous pressure is applied on the top face.

\section{Results and Discussion}

\subsection{Determination of Sandwich Material Properties}

Figure 10 shows the results of the tensile tests for material property extraction. Reproducible behaviour regarding the elastic properties can be observed. Young's modulus of the base material $E_{s}$ can be derived to $E_{s} \approx 60.000 \mathrm{MPa}$ and yield strength to $\sigma_{Y} \approx 170 \mathrm{MPa}$. Elongation at the breakpoint and ultimate strength show different values, which is probably attributed to the strong influence of the manufacturing process. Compared to the manufacturer's datasheet, Young's modulus and yield double-point are in a tolerable range, whereas elongation at the breakpoint and ultimate strength are much lower than specified. The more brittle behaviour is mainly attributed to the thickness of the structures. Material properties in the datasheet are extracted from thick, solid structures, whereas in this study, thin structures are investigated.

Using Eqs. (2) and (3) and the extracted material data, the following material and sandwich properties are derived (Table 5).

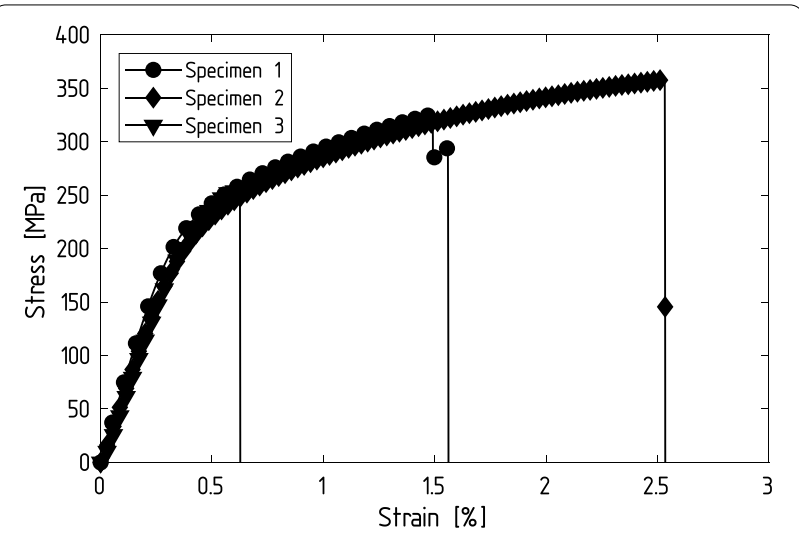

Figure 10 Stress-strain curves of AlSi10Mg tensile tests on AM-sandwich structures according to E-DIN-EN-ISO 6892-1:2019-10

Table 5 Resulting sandwich properties

\begin{tabular}{llll}
\hline Desription & Symbol & Value & Unit \\
\hline Base material & & & \\
Young's modulus & $E_{S}$ & 60.000 & $\mathrm{MPa}$ \\
Poisson ratio & $v_{S}$ & 0.3 & - \\
Yield strength & $\sigma_{Y}$ & 270 & $\mathrm{MPa}$ \\
Rel. density & $\bar{\rho}$ & 0.13884 & - \\
Effective elastic properties & & \\
& $E_{11}$ & 60.000 & $\mathrm{MPa}$ \\
& $E_{22}$ & 60.000 & $\mathrm{MPa}$ \\
& $E_{33}$ & 2082.6 & $\mathrm{MPa}$ \\
& $G_{12}$ & 23076.9 & $\mathrm{MPa}$ \\
& $G_{13}$ & 1041.3 & $\mathrm{MPa}$ \\
& $G_{23}$ & 1041.3 & $\mathrm{MPa}$ \\
\hline
\end{tabular}

\subsection{Analytical Failure Mode Calculation}

Throughout the study, two test strategies are used to characterise the delamination behaviour of $\mathrm{AM}$ 
sandwich structures: out-of-plane tensile tests and climbing drum peel tests. Furthermore, the tests are supported by numerical FE calculations to better understand the failure mechanisms. First, the main failure mechanisms for the core structure are determined. Here, failure can happen in two different zones: at the connection double-point or in the strut itself. Considering one unit cell with pinned struts, as shown in Figure 2, a load in 3-direction (out-of-plane) results in an axial strut force of:

$$
F_{s}=\frac{1}{4} \frac{F_{3}}{\sin (\omega)} .
$$

The failure zone is only a function of the strut angle $\omega$. This can be derived by comparing the strut stress with the stress in the connection surface. Under a tension load in 3-direction $F_{3}$, the resulting stresses in the struts $\sigma_{s}$ and the stress in the connection $\sigma_{c}$ can be calculated as followed:

$$
\begin{aligned}
\sigma_{s} & =\frac{F_{3}}{4 \pi d^{2} \sin (\omega)}, \\
\sigma_{c} & =\frac{F_{3}}{\arctan (\csc (\omega)) \csc (\omega) d^{2}} .
\end{aligned}
$$

Under the condition that the struts are slender, and by this, no bending influence has to be considered, simple assumptions can be derived for the predicted failure mechanism. For $\sigma_{s}=\sigma_{c}$ a critical value $\omega=35.26^{\circ}$ results. For lower angles, the struts will fail before the connection. For larger angles, the connection will fail before the struts. This relation is shown in Figure 11. In the following sections, the experimental setup and the expected, analytically determined values are shown.

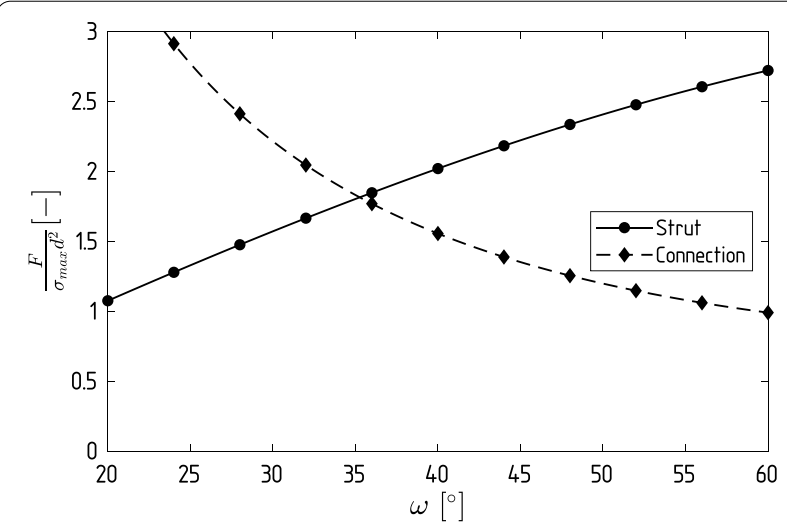

Figure 11 Failure in struts and connection as a function of relative angle
Taking into account the considerations for the lattice core made above, two different failure modes can occur for the structures used in this study: failure of the face sheet, and failure at the connection between the face sheets and the core. Assuming an ideal elastic - ideal plastic material behaviour, the stress distribution in the face sheet, due to a bending moment, can be assumed to be linear in the case of elastic material behaviour and constant (with different signs) in the case of plastic material behaviour. The maximum moment which can be transferred before face sheet failure can be calculated with $M_{\mathrm{el}}=R_{m} t_{f} b / 6$ in the elastic case and $M_{\mathrm{pl}}=R_{m} t_{f} b / 4$ in the plastic case. Since ductile materials fail in the plastic area, the plastic case will be used. Figure 12 shows a submodel for two rows of the sandwich structure. The applied bending moment results in reaction forces $F_{R}=M / a$ at the connection double-points if pinned struts are assumed. The distance between two unit cells is $a$. By this, a general equation to avoid delamination is established:
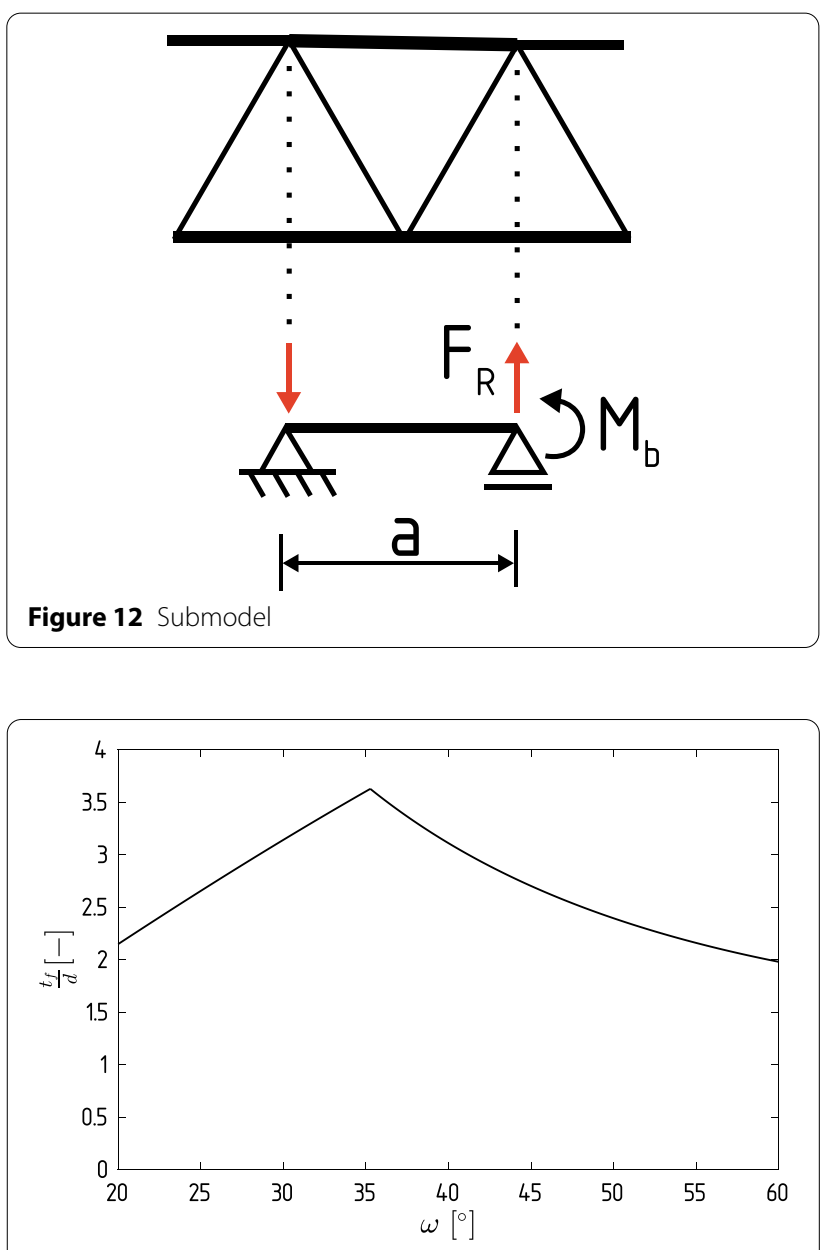

Figure 13 Critical ratio for $t_{f} / d$ to avoid delaminations 


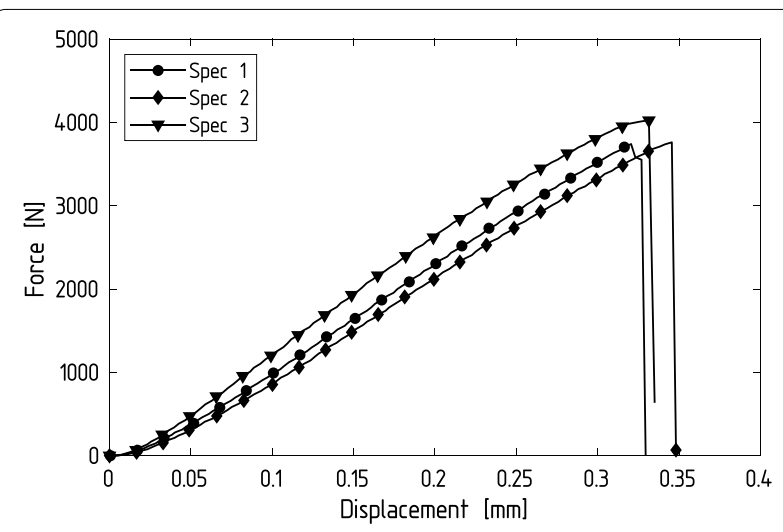

Figure 14 Load-displacement curve of out-of-plane tensile tests

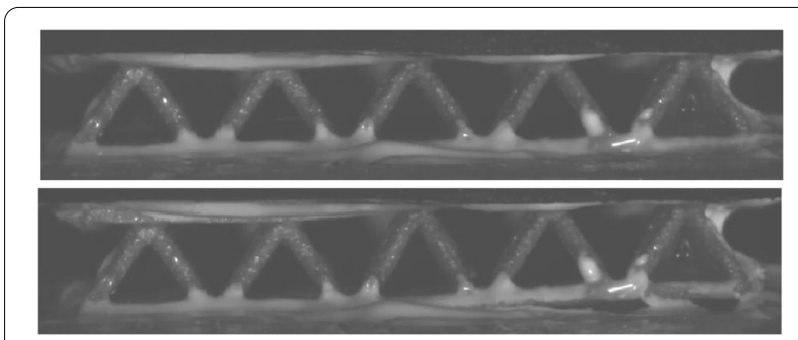

Figure 15 Pictures of one speciment during testing (Top: before load drop, Bottom: after load drop)

$$
\frac{t_{f}}{d}< \begin{cases}2 \sqrt{\pi \sin \omega}, & \omega \leq 35.26^{\circ} \\ 2 \sqrt{\arctan (\csc (\omega)) \csc (\omega)}, & \omega>35.26^{\circ}\end{cases}
$$

This relation is shown in Figure 13. If delaminations should be avoided, the ratio of $t_{f} / d$ should be lower than the in Figure 13 shown critical ratio. The critical value for $\omega$ is the value where the failure mode changes from strut failure to joint failure (see Figure 11). The ratio $t_{f} / d$ increases with increasing $\omega$ up to the critical value, after which it starts decreasing, as shown in Figure 13. Therefore, for $\omega=45^{\circ}, t_{f} / d<2.32$ should be complied to avoid delaminations.

\subsection{Out-of-Plane Tensile Test}

Figure 14 shows the results of the out-of-plane tensile tests. Additionally, Figure 15 shows a picture of one of the specimens right before failure and after failure. The results show slight differences regarding the stiffness, which can be attributed mainly to manufacturing tolerances. The tolerances, specified to be $\pm 100 \mu \mathrm{m}$, affect the strut diameters and therefore influence the stiffness quadratically (see Eqs. (1) and (2)). The failure load of all three tested specimens lies between $3.8-4 \mathrm{kN}$. No failure in the core structure was detected. For all specimens, the adhesive fails before the core breaks. All failure loads are smaller than expected, which could be attributed to an irregular moistened adhesive surface. Nevertheless, from the measured loads, it follows that the connection is at least as strong as the strength of the material (Figure 10). Assuming the connection surface of one unit cell to be, as specified in Figure $3, A_{c} \approx 0.34 \mathrm{~mm}^{2}$ and the resulting total connection surface $A_{C}=n \times A_{c}=8.5 \mathrm{~mm}^{2}$. This results in a connection strength of at least $\sigma_{u}>440 \mathrm{MPa}$, which is not a reasonable value. Due to these circumstances, the face sheets are removed from the core structure, and the bonding surfaces are measured using optical microscopy. Although the diameter of the struts is between 0.5 and $0.6 \mathrm{~mm}$, the measured connection surfaces are clearly above the theoretically calculated value. The measured bonding surfaces lie partly at around $2 \mathrm{~mm}^{2}$. Assuming such a single cell bonding surface, a total bonding surface of $A_{C}=50 \mathrm{~mm}^{2}$ would result, leading to a stress of $\sigma_{c} \approx 80 \mathrm{MPa}$ in the connection. This explains why the adhesive fails and not the connection between the faces and the core.

Based on the numerical FE simulations, the first zone to undergo full plastic deformation is the joint between the face and the struts, as shown in Figure 16. This is also the expected critical zone according to Eqs. (7) and (8).

\subsection{Climbing Drum Peel Test}

Figure 17 shows the force-displacement curves of the climbing drum peel tests. Furthermore, in Figure 18 the measured force is related to the specific width $\tilde{b}$ (Figure 7), to avoid that results are misinterpreted by different geometries. Additionally, Figure 19 shows a picture of specimen 1 and Figure 20 of specimen 3 after testing. Both specimens show a failure in the face sheets. Since the face sheets fail before they have rolled up sufficiently, the evaluation according to DIN 53295 cannot be performed. Nevertheless, it can be followed that no delamination will occur with the used geometry. For specimen 3 , the number of cells in one row (in the rolling axis) was reduced. The face sheet did not fail immediately, and therefore, the evaluation for the peel moment can be done according to DIN 53295 to $M_{\text {peel }}=279.62 \mathrm{~N} \cdot \mathrm{mm} /$ $\mathrm{mm}$. However, the face sheet did not delaminate. Instead, it broke parallel to the cell row in rolling direction (see Figure 20), and by this, the result is not meaningful. 


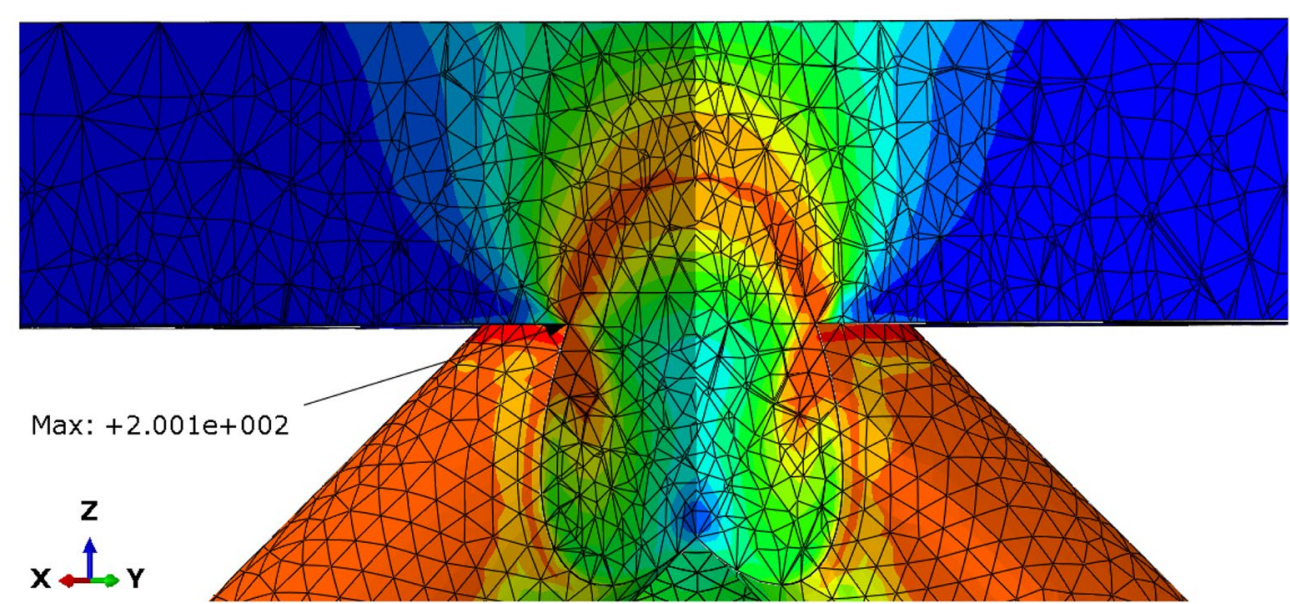

Figure $\mathbf{1 6}$ Section view at the middle joint

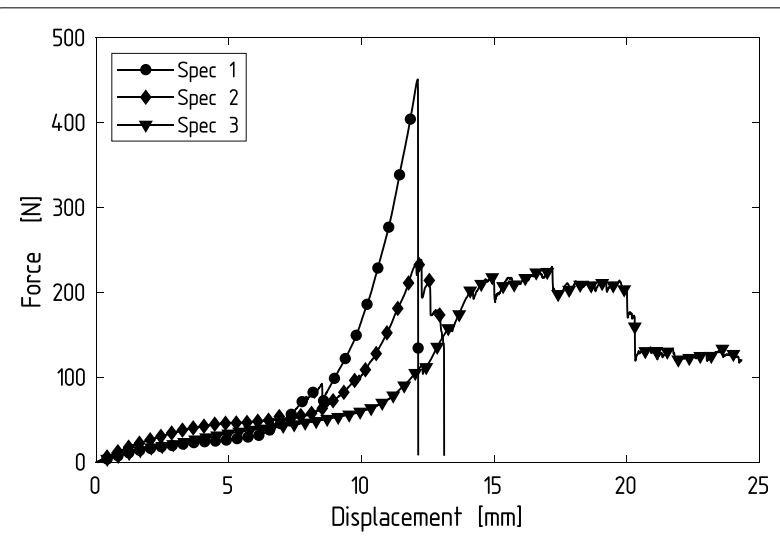

Figure 17 Force-displacement curve for the climbing drum peel test

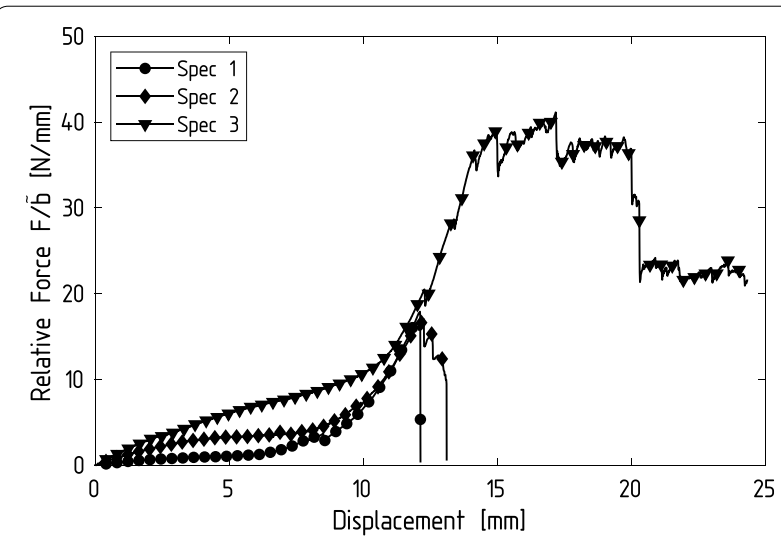

Figure 18 Relative force-displacement for the climbing drum peel test

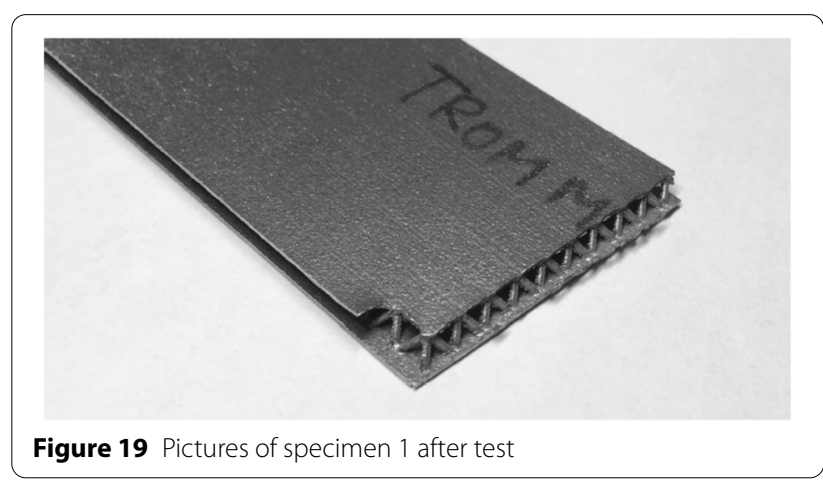

Based on the numerical FE simulations, the first zone to undergo full plastic deformation is the face sheet, as shown in Figure 21. This is also the expected critical zone according to Eq. (9) since $t_{f} / d=1<3.2$. In the second simulation, with $t_{f}=2 \Rightarrow t_{f} / d=4>3.2$, the connection is expected to fail (Figure 22), as seen in the stresses of Figure $9 \mathrm{~b}$. This corresponds to the simplified model presented in Eq. (9).

\section{Conclusions}

This study has investigated the delamination behaviour of fully additive manufactured sandwich structures with pyramidal lattice grid core. Simplified formulas were derived to estimate the geometry needed to avoid delamination failure and validated with experimental tests and numerical simulations. The analytical approaches show that delaminations can be avoided by considering critical values for the ratio between face sheet thickness and strut diameter. These ratios are 


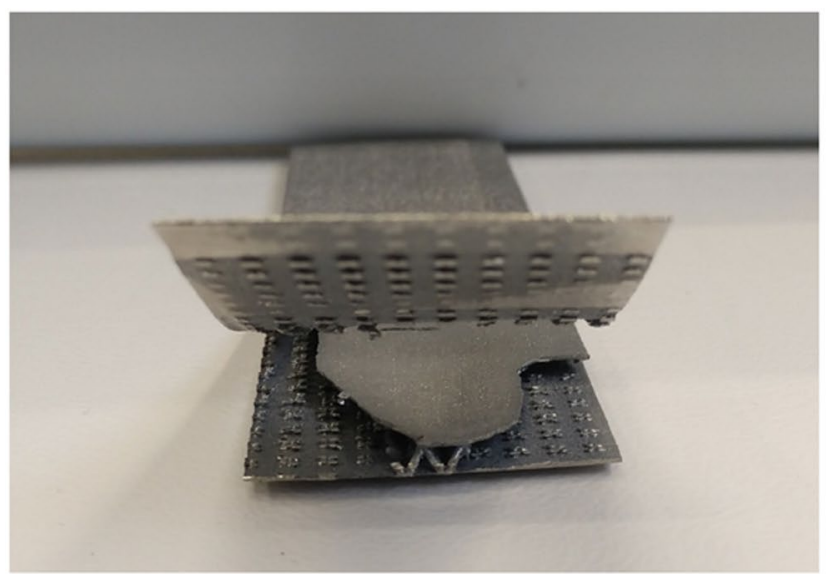

(a)

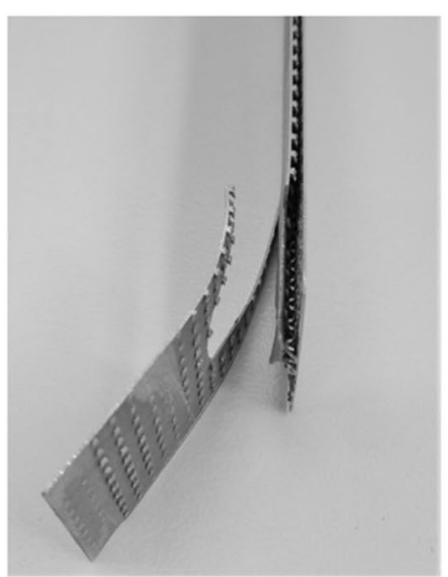

(b)

Figure 20 Pictures of specimen 3 after test

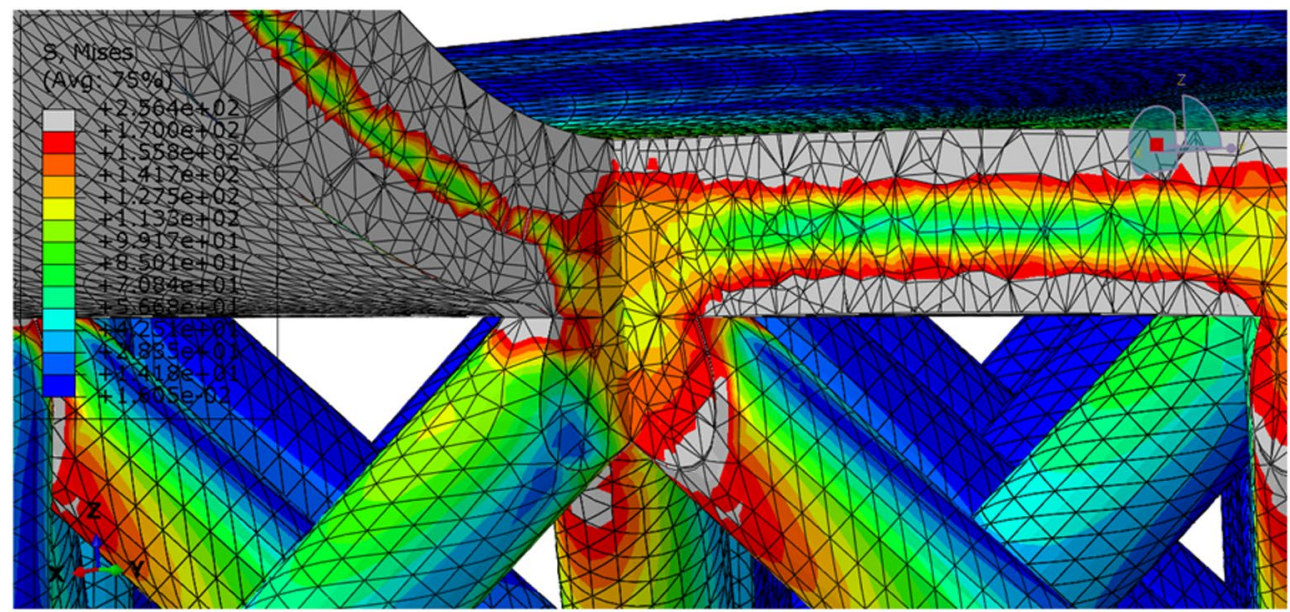

Figure 21 Section view of the specimen model at the middle joint of the outer row of cells. Zones with plastic deformation are marked grey

mainly dependent on the strut angle. By choosing an adequate set of geometric parameters, delaminations can be avoided. Since there were no studies related to the delamination behaviour of those structures, the results presented in this paper can be used as guidelines to design delamination free sandwich structures with pyramidal lattice grid core. In future studies, the models will be expanded to assume an inhomogeneous stress distribution on the joints and struts. 


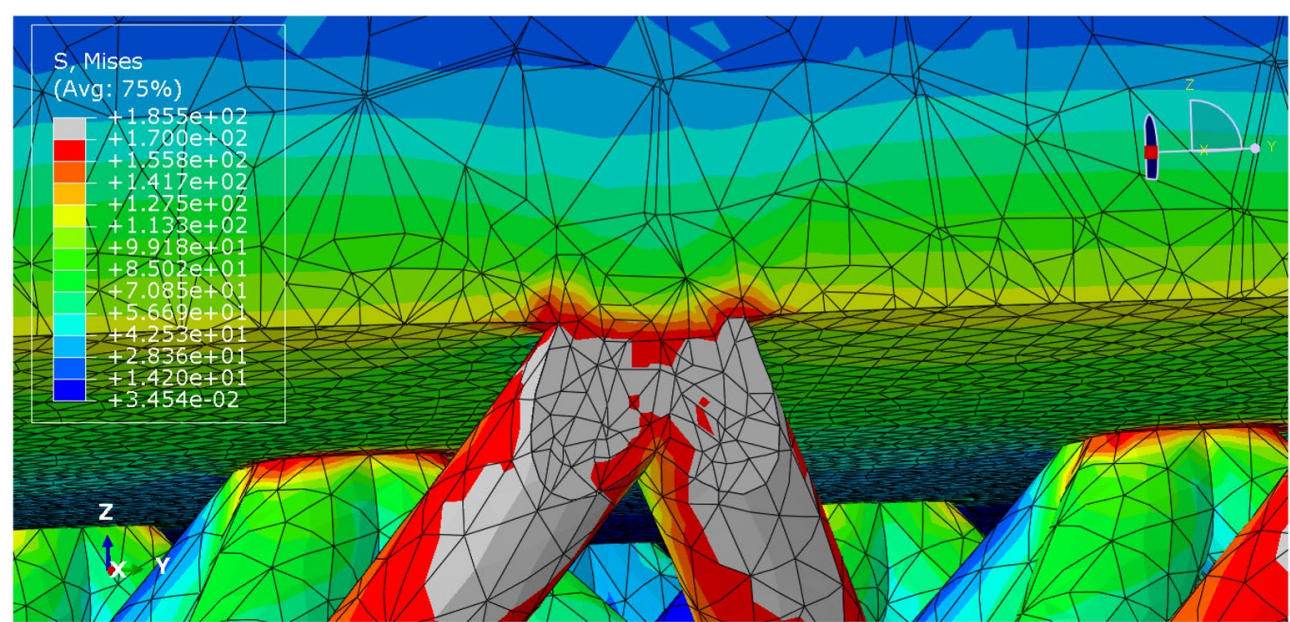

Figure 22 Section view of the model with thick faces at the middle joint of the outer row of cells. Zones with plastic deformation are marked grey

\section{Acknowledgements}

Not applicable.

\section{Authors' Contributions}

$\mathrm{MN}$ and JB have contributed equally to this article and where in charge for the whole trial. NMR and KUS supported with their extensive experience and gave advices on the manuscript. All authors read and approved the final manuscript.

\section{Funding}

Part of this work was supported by the German Federal Ministry for Economic Affairs and Energy (BMWi) (Grant No. 20E1713B).

\section{Availability of Data and Materials}

The datasets used and/or analysed during the current study are available from the corresponding author on reasonable request.

\section{Competing Interests}

The authors declare that they have no competing interests.

Received: 26 March 2021 Revised: 28 October 2021 Accepted: 3 November 2021

Published online: 04 December 2021

\section{References}

[1] F Froes, R Boyer, eds. Additive manufacturing for the aerospace industry. Elsevier, Amsterdam, Netherlands, 2019.

[2] R Liu, Z Wang, T Sparks, et al. Aerospace applications of laser additive manufacturing. In: Laser Additive Manufacturing, Elsevier, 2017, 3: 351-371.

[3] S Mohd Yusuf, S Cutler, N Gao. Review: The impact of metal additive manufacturing on the aerospace industry. Metals, 2019, 9(12): 1286.

[4] J C Najmon, S Raeisi, A Tovar. Review of additive manufacturing technologies and applications in the aerospace industry. In: Additive Manufacturing for the Aerospace Industry, Elsevier, 2018, 69: 7-31.

[5] A Uriondo, M Esperon-Miguez, S Perinpanayagam. The present and future of additive manufacturing in the aerospace sector: A review of important aspects. Proceedings of the Institution of Mechanical Engineers, Part G: Journal of Aerospace Engineering, 2015, 229(11): 2132-2147.

[6] C Cai, C Radoslaw, J Zhang, et al. In-situ preparation and formation of tib/ ti-6al-4v nanocomposite via laser additive manufacturing: Microstructure evolution and tribological behavior. Powder Technology, 2019, 342(19): 73-84.
[7] C Cai, XWu, W Liu, et al. Selective laser melting of near-alpha titanium alloy ti-6al-2zr-1mo-1v: Parameter optimization, heat treatment and mechanical performance. Journal of Materials Science \& Technology, 2020, 57: 51-64.

[8] M E Orme, M Gschweitl, M Ferrari, et al. Additive manufacturing of lightweight, optimized, metallic components suitable for space flight. Journal of Spacecraft and Rockets, 2017, 54(5): 1050-1059.

[9] J Bühring, M Nuño, K U Schröder. Additive manufactured sandwich structures: Mechanical characterization and usage potential in small aircraft. Aerospace Science and Technology, 2021, 111(30-31): 106548.

[10] B Aslan, A R Yıldiz. Optimum design of automobile components using lattice structures for additive manufacturing. Materials Testing, 2020, 62(6): 633-639.

[11] A Borrelli, G D'Errico, C Borrelli, et al. Assessment of crash performance of an automotive component made through additive manufacturing. Applied Sciences, 2020, 10(24): 9106.

[12] J C Vasco. Additive manufacturing for the automotive industry. In: Additive Manufacturing, Elsevier, 2021, 15: 505-530.

[13] J Bühring, M Voshage, J H Schleifenbaum, et al. Influence of degradation product thickness on the elastic stiffness of porous absorbable scaffolds made from an bioabsorbable zn-mg alloy. Materials, 2021, 14(20): 6027.

[14] W Frank, J Lucas, M Wolfgang, et al. Open-porous biodegradable magnesium scaffolds produced by selective laser melting for individualized bone replacement. Frontiers in Bioengineering and Biotechnology, 2016, 4.

[15] A Kopp, T Derra, M Müther, et al. Influence of design and postprocessing parameters on the degradation behavior and mechanical properties of additively manufactured magnesium scaffolds. Acta Biomaterialia, 2019, 98: 23-35.

[16] Y Li, H Jahr, J Zhou, et al. Additively manufactured biodegradable porous metals. Acta Biomaterialia, 2020, 115: 29-50.

[17] Y Li, J Zhou, P Pavanram, et al. Additively manufactured biodegradable porous magnesium. Acta Biomaterialia, 2018, 67: 378-392.

[18] X Liu, C Zhao, X Zhou, et al. Microstructure of selective laser melted alsi10mg alloy. Materials \& Design, 2019, 168(S3): 107677.

[19] U Tradowsky, J White, R M Ward, et al. Selective laser melting of alsi10mg: Influence of post-processing on the microstructural and tensile properties development. Materials \& Design, 2016, 105: 212-222.

[20] P Yang, L A Deibler, D R Bradley, et al. Microstructure evolution and thermal properties of an additively manufactured, solution treatable alsi10mg part. Journal of Materials Research, 2018, 33(23): 4040-4052.

[21] D Martínez-Maradiaga, O V Mishin, K Engelbrecht. Thermal properties of selectively laser-melted alsi10mg products with different densities. Journal of Materials Engineering and Performance, 2020, 29(11): 7125-7130. 
[22] W Li, S Li, J Liu, et al. Effect of heat treatment on alsi10mg alloy fabricated by selective laser melting: Microstructure evolution, mechanical properties and fracture mechanism. Materials Science and Engineering: A, 2016, 663: 116-125.

[23] N Read, W Wang, K Essa, et al. Selective laser melting of alsi10mg alloy: Process optimisation and mechanical properties development. Materials \& Design (1980-2015), 2015, 65: 417-424.

[24] Y Feng, H Qiu, Y Gao, et al. Creative design for sandwich structures: A review. International Journal of Advanced Robotic Systems, 2020, 17(3): 172988142092132.

[25] N Wicks, J W Hutchinson. Optimal truss plates. International Journal of Solids and Structures, 2001, 38(30-31): 5165-5183.

[26] N Wicks, JW Hutchinson. Performance of sandwich plates with truss cores. Mechanics of Materials, 2004, 36(8): 739-751.

[27] L J Gibson. Cellular solids. Cambridge Univ. Press, Cambridge, 2001.

[28] M F Ashby. The properties of foams and lattices. Philosophical Transactions of the Royal Society A: Mathematical, Physical and Engineering Sciences, 2006, 364(1838): 15-30.

[29] V S Deshpande, N A Fleck. Collapse of truss core sandwich beams in 3-point bending. International Journal of Solids and Structures, 2001, 38(36-37): 6275-6305.

[30] V S Deshpande, N A Fleck, M F Ashby. Effective properties of the octettruss lattice material. Journal of the Mechanics and Physics of Solids, 2001, 49(8): 1747-1769.

[31] A G Evans, JW Hutchinson, M F Ashby. Multifunctionality of cellular metal systems. Progress in Materials Science, 1998, 43(3): 171-221.

[32] A G Evans, JW Hutchinson, N A Fleck, et al. The topological design of multifunctional cellular metals. Progress in Materials Science, 2001, 46(3-4): 309-327.

[33] Chiras S Mumm, A G Evans, N Wicks, et al. The structural performance of near-optimized truss core panels. International Journal of Solids and Structures, 2002, 39(15): 4093-4115.

[34] F W Zok, S A Waltner, Z Wei, et al. A protocol for characterizing the structural performance of metallic sandwich panels: application to pyramidal truss cores. International Journal of Solids and Structures, 2004, 41 (22-23): 6249-6271.

[35] D Zenkert, O Schubert, M Burman. Fracture initiation in foam-core sandwich structures due to singular stresses at corners of flawed butt joints. Mechanics of Advanced Materials and Structures, 1997, 4(1): 1-21.

[36] D Zenkert. Fatigue of foam core sandwich beams - 1: undamaged specimens. International Journal of Fatigue, 1997, 19(7): 551-561.

[37] Shipsha, Burman, Zenkert. Interfacial fatigue crack growth in foam core sandwich structures. Fatigue \& Fracture of Engineering Materials \& Structures, 1999, 22(2): 123-131.

[38] J L Grenestedt. Development of a new peel-stopper for sandwich structures. Composites Science and Technology, 2001, 61(11): 1555-1559.

[39] CWonderly, J Grenestedt. Dynamic performance of a peel stopper for composite sandwich ship structures. Journal of Composite Materials, 2004, 38(10): 805-831.

[40] B Lascoup, Z Aboura, K Khellil, et al. On the mechanical effect of stitch addition in sandwich panel. Composites Science and Technology, 2006, 66(10): 1385-1398.

[41] P Potluri, E Kusak, T Reddy. Novel stitch-bonded sandwich composite structures. Composite Structures, 2003, 59(2): 251-259.

[42] J Jakobsen, E Bozhevolnaya, O T Thomsen. New peel stopper concept for sandwich structures. Composites Science and Technology, 2007, 67(15-16): 3378-3385. 